\title{
Deliberative Argumentation for Smart Environments
}

\author{
Juan Carlos Nieves, Esteban Guerrero, Jayalakshmi Baskar, Helena Lindgren \\ Department of Computing Science \\ Umeå University \\ SE-901 87, Umeå, Sweden \\ \{jcnieves,esteban,jaya,helena\}@cs.umu.se
}

\begin{abstract}
In this paper, an argumentation-based deliberative approach for fusing contextual information obtained from heterogeneous sources using a multi-agent system is introduced. The system is characterized by three different agents: an Environment Agent, an Activity Agent and a Coach Agent. These agents consider data from heterogenous sources of data. As a method for aggregating data and supporting decision-making, so-called agreement rules are instrumental in the argumentationbased deliberative method. The aggregation rules will be associated to specific beliefs related to the services of each agent.
\end{abstract}

\section{Introduction}

As a result of the daily activities of humans, there can be a vast and increasing volume of a variety of data that is collected from different sources, e.g., sensors from smart environments. The data is typically represented using a variety of formats, e.g., relational databases, rule-based knowledge bases, etc. Moreover, these data sources are consulted by using different query engines, in order to serve the human with useful information. Consequently, providing services, such as support for decision-making by synthesizing the relevant sources of data, represents a fundamental challenge in information management.

In this paper, an argumentation-based deliberative approach for fusing contextual information from heterogeneous sources using a multi-agent system is introduced. Deliberation dialogues aim at reasoning for deciding upon what action to make (also called practical reasoning in literature) and have been explored by several authors in the argumentation literature [1|2|3|8]. The argumentation-based deliberative approach introduced in this paper, is motivated by the design and construction of As-A-Pal, a smart home environment functioning as a part of the As-A-Pal architecture initially presented in [9]. The As-A-Pal project aims at developing an agent-based assessment and intervention infrastructure, where personalized interventions are provided, which can be viewed as services. Therefore, our multi-agent system is described in terms of the services supported by AsA-Pal.

The paper presents the following two major contributions: 1) a multi-agent approach designed to fuse contextual information from heterogeneous sources; and 2) an argumentationbased deliberative method based on argument inquiry dialogues [3], Well-Founded Semantics (WFS) [4] and agreement rules. The materialization of the multi-agent approach is based on three software agents: an Environment Agent, an Activity Agent and a Coach Agent. These three agents have different goals and different capabilities; however, they are 
collaborative in order to provide services to a user in smart environments. The design of these agents follows the conceptual models of Activity Theory [7]. The inquiry dialogues support both data aggregation and decision making from heterogenous sources of data. The deliberative method is based on both knowledge bases expressed in terms of logic programs with negation as failure and WFS [4]. We show that our approach is sound w.r.t. the inference of WFS. Moreover, we show that deciding whether an agreement rule is committed is decidable in polynomial time.

The article is organized as follows: Section 2 introduces our suggested multi-agent approach. Section 3 introduces our argumentation-based deliberative method. In the last section, our conclusions and future work are presented.

\section{A Multi-Agent Systems for providing intelligent services}

In this section, we present the first contribution of this paper, which is a multi-agent approach designed to deliver personalized services in a smart environment.

Since human activity performance is complex, we apply activity-theoretical models for capturing motives, goals, composite actions, the role of tools, and the levels of complexity of actions [7]. The use of activity-theoretical models is justified by the following three reasons. Firstly, Activity Theory emphasizes that human activity is affected by the environment, and dependent on the availability and the characteristics of tools, which enable and mediate activity. Consequently, the physical environment is taken into consideration, when a human actor is moving around, finding tools to use in activities. Secondly, activity is composed of actions in an hierarchy of complexity, which is dynamic, and puts challenges on technology aimed at recognizing and evaluating activity in an ambient assisted living environment [7]. For instance, the human can conduct her breakfast routine in different ways, even cook her porridge in different ways. The selection of procedure depends on several factors, some which the human is aware of, and some selections are done automatically, without thinking. For a support system to know and decide if, or at which point, the system should interfere for giving support is a non-trivial task. Thirdly, Activity Theory emphasizes the changing nature of activity and human ability to perform activity, driven by motives, goals, challenges and focus shifts, which in turn drives development [7]. The concept zone of proximal development (ZPD) is applied, where a human Actor is expected to be able to perform an activity with the assistance of a more skillful peer, until the autonomy is reached. In our scenario, the intervention in the form of the As-A-Pal system functions as the more skillful peer, for the purpose to improve activity performance. These three activity-theoretical perspectives are captured by the so called Environment Agent, Activity Agent and Coach Agent, see Figure 1 .

The agents have been partially implemented in the As-A-Pal smart environment. They supplement the Domain Agent, previously introduced [9], which has the role of a domain expert in our multi-agent systems. However, in this work, we focus on the other three agents. The three agents are human-centered, i.e. they take the human Actor as the starting point while providing the personalized services. As-A-Pal is a smart environment where the acronym As-A-Pal refers to Agent-Supported Assessment for Adaptive and Personalized Ambient Assisted Living. As-A-Pal also refers to "like a friend", an artificial companion that knows the immediate needs of the human actor, her preferences, priorities and 


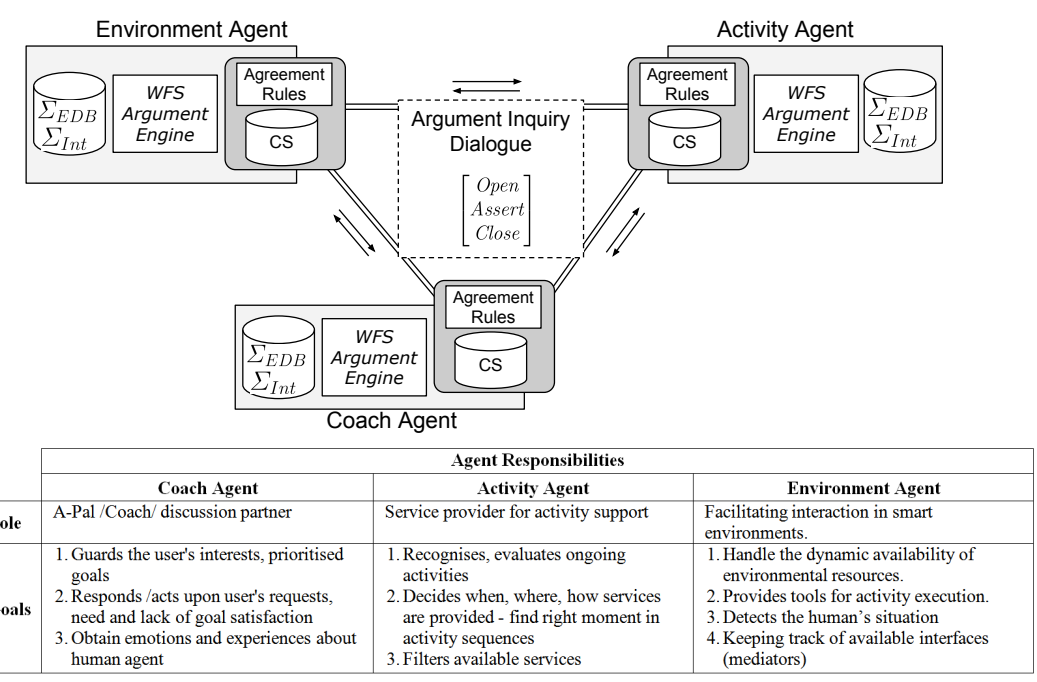

Fig. 1. Interaction between agents and their goals.

abilities, so that adaptive and personalized services tailored to the current context can be provided.

\section{A Deliberative Argumentation Approach}

In Section 2, a multi-agent architecture was introduced. One of the main issues to deal with in this architecture, is to reach agreements between its agent in order to select the best service to offer to an end-user. In this section, an argumentation approach is presented, which manages agreements between the As-A-Pal architecture's agents. This argumentation approach will be basically an operational implementation of an deliberation dialogue. A deliberation dialogue is characterized as a dialogue occurring when two or more parties aim to agree on an action in some situation. To implement deliberation dialogues between the As-A-Pal's agents, we provide each agent with a set of so-called agreement rules. An agreement rule is basically a consensus in which the different participant of a deliberation dialogue agree. Agreement rules will be associated to specific goals related to the services of the As-A-Pal architecture. Hence, an agreement rule will be defined as follows.

Let us start presenting the following notation: given a logic-based theory $T, \mathcal{L}_{T}$ denotes the set of atoms which appears in $T$.

Definition 1. An agreement rule is of the form:

$$
\alpha: a_{0} \leftarrow a_{1}, \ldots, a_{n}
$$

in which $\alpha \in \mathbb{N}, a_{i}(0 \leq i \leq n)$ is an atom such that for each $a_{i}(1 \leq i \leq n)$ either exists an agent $\mathrm{Ag}$ such that its logic-based knowledge base is $\Sigma$ and $a_{i} \in \mathcal{L}_{\Sigma}$ or $a_{i} \in \mathcal{L}_{A R}$ such that $A R$ is a set of agreement rules, and $a_{0} \neq a_{i}(1 \leq i \leq n)$. 
In the context of the As-A-Pal architecture, the head of an agreement rule, a.i. $a_{o}$, will be associated to a particular belief of the As-A-Pal architecture. For instance, this belief can be a service suitable for the end user. This means that by considering the trueness of an agreement rule different agents will agree on a particular service for a user.

According to Definition 1, each agreement rule has a natural number attached. This natural number denotes a preference level. In the As-A-Pal architecture, the preference levels are managed by the Coach Agent by considering user-satisfiability.

Example 1. Let us consider a couple of agreement rules of the As-A-Pal smart environment as illustration:

\begin{tabular}{|c|c|}
\hline$A R 1$ & $\begin{aligned} \alpha_{1}: \text { service }(\text { reminder }, Z, M) \leftarrow \text { optimal_mediator }(Z, M), \neg \text { past_activity }(Z, \\
\text { takenpills }), \text { requested_service }(Z, \text { reminders }) .\end{aligned}$ \\
\hline$\overline{A R 2}$ & $\begin{aligned} &(Z, M) \leftarrow \text { mediator }(M), \text { is_near }(M, Z), \text { current_activity }(Z, X), \\
& \text { acivity_object }(M, X) .\end{aligned}$ \\
\hline
\end{tabular}

In these agreement rules, we are assuming that predicates such as mediator $(M)$ and $i s \_n e a r(M, Z)$ belong to the knowledge base of the environment agent. Moreover, the predicates current_activity $(Z, X)$ belongs to the knowledge base of the activity agent. Therefore, the agents have to interact in order to decide if a given agreement rule holds true in a given state of the As-A-Pal system.

The general idea of our approach is to consider an argument inquiry dialogue in order to validate the trueness of a given agreement rule. If an agreement rule holds true in an given state of the As-A-Pal architecture, then the head of the given agreement rule holds the trueness of a particular believe in the whole As-A-Pal system.

Inspired by [3], our argument inquiry dialogues are based on three basic moves: open: $\langle x$, open, dialogue $(a i, \gamma)\rangle$, assert: $\langle x$, assert,$\langle S, a\rangle\rangle$ and close: $\langle x$, close, dialogue $(a i, \gamma)\rangle$ in which $x$ denotes an agent, $\langle S, a\rangle$ is an argument, $\gamma$ denotes an agreement rule and ai means "argument inquiry dialogue". It should be noted that the format of these moves are not exactly the same as the ones introduced by [3]. Our moves are applied to only the argument inquiry dialogue type, which can be opened only by applying an agreement rule as a topic. This means that the nested process between argument inquiry dialogues is only induced by agreement rules. Moreover, the arguments suggested by assert-moves will be constructed based on the deductive arguments introduced in [6]. We have implemented an argumentation engine, which constructs these arguments from a logic program [6] Given a logic program $\Sigma, \mathcal{A}_{\Sigma}$ denotes the set of arguments built from $\Sigma$.

From hereon, we apply the definition of dialogue introduced in [3]. $D_{r}^{t}$ denotes a dialogue which is a sequence of moves $\left[m_{r}, \ldots, m_{t}\right]$ involving a set of participants $\mathcal{I}$, where $r, t \in \mathbb{N}$. As in [3], we apply the restriction that a dialogue terminates whenever all the participants of a dialogue have made a close move in a consecutive form. In addition, it is allowed to open another dialogue without terminating the ongoing dialogue, which allows us to manage multi-nested dialogues.

In the following we present the protocol of an argument inquiry dialogue as a sequence of general steps. Let $\mathcal{I}$ be the finite set of participants of a dialogue. We identify each agent from $\mathcal{I}$ by a natural number this means that $\mathcal{I}=1, \ldots, n$ such that $i=\left\langle\Sigma^{i}, A R^{i}, C S^{i}\right\rangle$ in which $\Sigma^{i}$ denotes the knowledge base of agent $i, A R^{i}$ denotes a set of agrement rules

\footnotetext{
${ }^{1}$ This argumentation engine can be downloaded from: http://esteban-guerrero. tumblr.com/argengine
} 
which belongs to agent $i$ and $C S^{i}$ denotes a commitment store of agent $i . \Sigma^{i}$ and $C S^{i}$ are basically extended normal logic programs. As it is done in [3], a dialogue is attached by a query store. Hence, an argument inquiry dialogue works as follows:

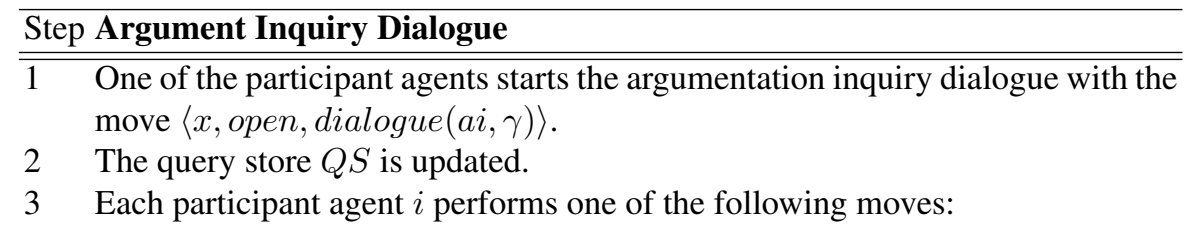

1. $\langle i$, assert,$\langle S, a\rangle\rangle$ if $\langle S, a\rangle \in \mathcal{A}_{\Sigma}, a \in Q S$ in which $\Sigma=\Sigma^{i} \cup$ $\bigcup_{j \in \mathcal{I} \text { and } i \neq j} C S^{j}$ and none of the participants have asserted the argument $\langle S, a\rangle$ in the dialogue before. The commitment store of the agent $i$ is updated.

2. 〈i,open, dialogue $\left.\left(a i, a_{0} \leftarrow a_{1}, \ldots, a_{n}\right)\right\rangle$ if $a_{0} \in Q S, \alpha: a_{0} \leftarrow$ $a_{1}, \ldots, a_{n} \in A R^{i}$ and there is no previous open move in the dialogue with $a_{0} \leftarrow a_{1}, \ldots, a_{n}$ as its topic. The dialogue go to Step 1 in a recursive way.

3. $\langle i$, close, dialogue $(a i, \gamma)\rangle$ if the agent $i$ is unable to perform one of the previous steps.

There are formal conditions w.r.t. well-formed argument inquiry dialogues, which basically argue that all the moves extend an initial dialogue and all the participants of the dialogue have the opportunity to perform a move (see [3] for the formal definitions).

Given an argument inquiry dialogue, its outcome is defined as follows:

Definition 2. Let $D_{r}^{t}$ be a well-formed argument inquiry dialogue. The outcome of $D_{r}^{t}$ is: Outcome $_{a i}\left(D_{r}^{t}\right)=\mathcal{A}_{\Sigma}$ such that $\Sigma=\bigcup_{i \in \mathcal{I}} C S^{i}$.

As we can see in Definition 2, the outcome of an argument inquiry dialogue is basically the set of arguments, which we can build from the commitment stores of all the participating agents.

In order to define when an agreement rule $\gamma$ is committed by a set of agents $\mathcal{I}$, let us introduce the concept of an agrement atom. Let $i=\langle\Sigma, A R, C S\rangle$ be an agent. $a \in \mathcal{L}_{A R}$ is called an agrement atom iff $a \notin \mathcal{L}_{\Sigma}$ and it does not exist an agent $j=\left\langle\Sigma^{j}, A R^{j}, C S^{j}\right\rangle$ such that $a \in \mathcal{L}_{\Sigma^{j}}$. This means that agreement atoms only appears in agreement rules.

We will say that an agreement rule $\gamma$ is committed by a set of agents $\mathcal{I}$ as follows:

Definition 3. Let $D_{r}^{t}$ be a well-formed argument inquiry dialogue involving a set of participant $\mathcal{I}$ and $m_{r}=\langle x$, open, dialogue $($ ai,$\gamma)\rangle$ such that $x \in \mathcal{I}$ and $\gamma=a_{0} \leftarrow a_{1}, \ldots, a_{n}$ is an agreement rule. $\gamma$ is a committed agreement rule by $\mathcal{I}$ w.r.t. $D_{r}^{t}$ if for each $a_{i}(1 \leq$ $i \leq n)$ one of the following conditions hold:

1. if $a_{i}$ is not an agreement atom, then $\left\langle S, a_{i}\right\rangle \in$ Outcome $_{a i}\left(D_{r}^{t}\right)$.

2. if $a_{i}$ is an agreement atom, then there exist a sub-well-formed argument inquiry dialogue $D_{q}^{j}$ such that $m_{q}=\left\langle\right.$ agent, open, dialogue $\left.\left(a i, a_{i} \leftarrow a_{1}, \ldots, a_{k}\right)\right\rangle$ and $a_{i} \leftarrow$ $a_{1}, \ldots, a_{k}$ is a committed agreement rule by $\mathcal{I}$ w.r.t. $D_{q}^{j}$. 
An important property of a committed agreement rule is that this rule holds true by the well-founded model of the resulting program of the join of all the commitment stores and agreement rules of the participating agents.

Theorem 1. Let $D_{r}^{t}$ be a well-formed argument inquiry dialogue involving a set of participant $\mathcal{I}$ and $m_{r}=\langle x$,open, dialogue $($ ai,$\gamma)\rangle$ such that $x \in \mathcal{I}, \gamma=a_{0} \leftarrow a_{1}, \ldots, a_{n}$ is an agreement rule. If $\gamma$ is a committed agreement rule by $\mathcal{I}$ w.r.t. $D_{r}^{t}$ iff $a_{i} \in T(1 \leq i \leq n)$ such that $\Sigma=\bigcup_{i \in \mathcal{I}} C S^{i} \cup A R^{i}$ and $W F S(\Sigma)=\langle T, F\rangle^{2}$

Another relevant property of a committed agreement rule is that deciding whether an agreement rule is committed is decidable in polynomial time.

Proposition 1. Let $\gamma$ be an agreement rule, $\mathcal{I}$ be a set of agents and $D_{r}^{t}$ be an argument inquiry dialogue. Deciding whether $\gamma$ is a committed agreement rule by $\mathcal{I}$ w.r.t. $D_{r}^{t}$ is decidable in polynomial time.

Since more than one agreement rule could hold committed in a given moment, we will consider the preference level of each agreement rule for selecting the best service, which the As-A-Pal architecture can provide to an end-user.

Definition 4. Let $\gamma_{a}=\alpha_{a}: a_{0} \leftarrow a_{1}, \ldots, a_{n}$ and $\gamma_{b}=\alpha_{b}:=b_{0} \leftarrow b_{1}, \ldots, b_{n}$ be committed agreement rule. $\gamma_{a}$ is preferred than $\gamma_{b}$ if $\alpha_{a}<\alpha_{b}$.

Whenever an agent has to take a decision about which service to provide, it will take the service supported by a preferred committed agreement rule. If there are two committed agreement rules with the same preference level, the agent will take one of the these committed agreement rules in a random way. We could think that taking a committed agreement rule in a random way could be a drawback of our approach. However, the preference level of each agreement rule will be updated according to the user satisfaction with the choice. In this setting, an agreement rule, which supports a service with a poor user satisfiability will decrease its preference level. The update of preference levels will be managed by the coach agent.

Example 2. Let us introduce a simple example of a well-formed argument inquiry dialogue. To reduce space in the presentation, we will associate some abbreviations to the grounded predicates of the knowledge bases of the agents:

\begin{tabular}{|c|c|c|c|}
\hline Predicate & Acronym & Predicate & Acronym \\
\hline service(reminder, rut, walker_tablet $)$ & $s 1$ & optimal_mediator(rut, walker_tablet) & om \\
\hline past_activity(rut, takenpills) & pa & requested_service(rut, reminders)) & rs \\
\hline mediator(walker_tablet) & $m w t$ & is_near(walker_tablet, rut) & $n w t$ \\
\hline current_activity(rut, walking) & $c w$ & activity_object(walker_tablet,walking) & $a w t$ \\
\hline current_activity(rut, takenpills) & ctp & & \\
\hline
\end{tabular}

In this example, we consider the agreement rules introduced in Example 11. Let us suppose that the agreement rule AR1 belongs to Coach Agent. AR1 aims to provide a reminder to the user about her medication. Before considering this reminder, Coach Agent needs to know whether the user has taken her medication. If not, Coach Agent needs to

\footnotetext{
${ }^{2} W F S(\Sigma)=\langle T, F\rangle$ denotes the 3-valued model of the logic program $\Sigma$. Informally speaking, $T$ denotes the set of atoms which are inferred true and $F$ denotes the set of atoms which are inferred false. See [4] for the formal definition of the well-founded model.
} 
know if there is an optimal mediator, e.g., a digital interface, near the user for delivering the reminder. To find an optimal mediator is a goal of Environment Agent. Hence, we assume that AR2 belongs to Environment Agent. To record activities performed by the user is a goal of Activity Agent. Given this scenario, Coach agent opens an argument inquiry dialogue: $\langle 1$, open, dialogue $(a i, s 1 \leftarrow o m, \neg p a, r s\rangle$. Table 1 illustrates the moves of the dialogue.

Table 1. An example of an argument inquiry dialogue. $m_{t}$ denotes the moves conducted by the agents. They are denoted as follows: Coach Agent (1), Environment Agent (2) or Activity Agent (3).

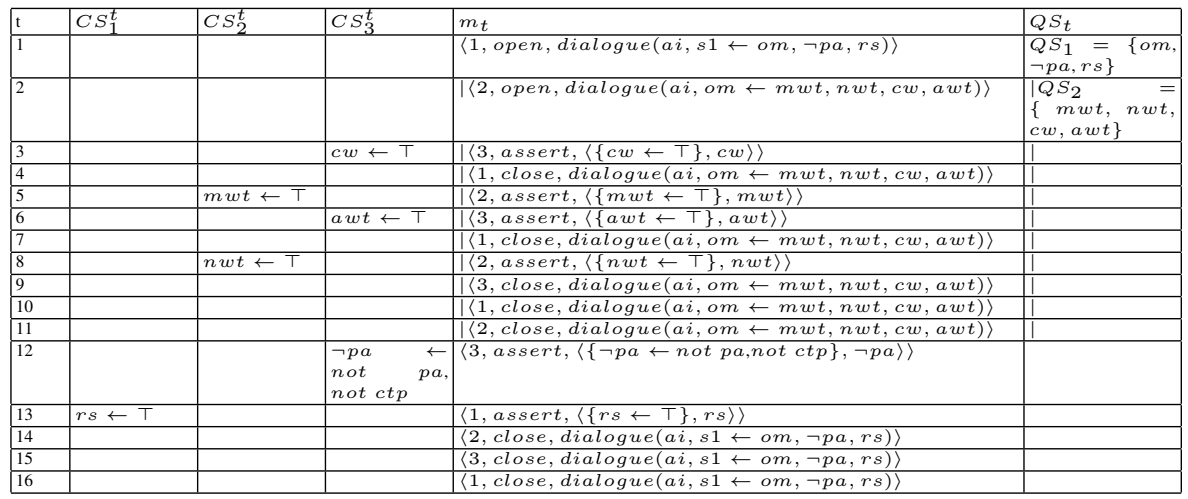

As can be observed in Table 1 . $A R 1$ is a committed agreement rule by all agents w.r.t. $D_{1}^{16}$. Moreover, $D_{1}^{16}$ is a well-formed argument inquiry dialogue. Let us observe that $D_{1}^{16}$ has the sub-dialogue $D_{2}^{11}$ which is also well-formed. Given that the atom service(reminder, rut, walker_tablet) is an agreement atom which suggests to deliver a reminder to the user Rut, Coach Agent can deliver a reminder to Rut in order to remind her to take her medication.

\section{Conclusions and Future Work}

In this paper, a multi-agent approach designed to fuse contextual information from heterogeneous sources in an ambient assisted living environment is presented. In particular, three agents have been introduced: the Environment Agent, the Activity Agent and the Coach Agent. The design of these agents follows conceptual models of Activity Theory, in order to capture the ambiguous and changing nature of human activity, and human preferences, needs, ability and motives. By introducing the concept of agreement rules, an argumentation-based deliberative method based on argument inquiry dialogues was also introduced. We show that our approach is sound w.r.t. the inference of the Well-Founded Semantics (WFS) (Theorem 1). Moreover, we show that deciding whether an agreement rule is committed is decidable in polynomial time (Proposition 1).

Our model of a deliberative process in smart environments was inspired by Black and Hunter's inquiry dialogue systems [3]. However, unlike Black and Hunter's inquiry dia- 
logue system [3], which is based on Defeasible Logic Programming for capturing knowledge and reasoning, we apply and implement logic programs with negation as failure and the WFS [4] for capturing knowledge and reasoning.

Our approach is conceptually close to the HERA project [10], which is an AAL system that provides specialized assisted living services for elderly people. From the knowledge representation point of view, our approach is similar to the one suggested by [11] in which the underlying mechanism for capturing the knowledge base relies on Extended Disjunctive Programs under the Answer Set Semantics [5].

In future work, we will focus on two main issues: 1) A combination of our argument inquiry dialogues with warrant inquiry dialogues in order to allow the agents to disagree with assert moves; 2) a complete implementation of our approach, using message-oriented middleware (MOMs) such as Data Distribution Service (DDS) for implementing argument inquiry dialogues.

Acknowledgement This research is partly funded by VINNOVA (Sweden's innovation agency) and the Swedish Brain Power.

\section{References}

1. L. Amgoud and H. Prade. Reaching agreement through argumentation: A possibilistic approach. In Principles of Knowledge Representation and Reasoning: Proceedings of the Ninth International Conference (KR2004), Whistler, Canada, June 2-5, 2004, pages 175-182. AAAI Press, 2004.

2. K. Atkinson, T. Bench-Capon, and D. Walton. Distinctive features of persuasion and deliberation dialogues. Argument and Computation, 4(2):105-127, 2012.

3. E. Black and A. Hunter. An inquiry dialogue system. Autonomous Agents and Multi-Agent Systems, 19(2):173-209, 2009.

4. A. V. Gelder, K. A. Ross, and J. S. Schlipf. The well-founded semantics for general logic programs. Journal of the ACM, 38(3):620-650, 1991.

5. M. Gelfond and V. Lifschitz. Classical negation in logic programs and disjunctive databases. New generation computing, 9(3-4):365-385, 1991.

6. E. Guerrero, J. C. Nieves, and H. Lindgren. Arguing through the Well-founded Semantics: an Argumentation Engine. Submitted to a journal, 2014.

7. V. Kaptelinin. Activity theory: Implications for human-computer interaction. In B. A. Nardi, editor, Context and Consciousness. Activity Theory and Human Computer Interaction, pages 103-116. MIT Press, 1996.

8. S. Kraus, K. P. Sycara, and A. Evenchik. Reaching agreements through argumentation: A logical model and implementation. Artif. Intell., 104(1-2):1-69, 1998.

9. H. Lindgren, D. Surie, and I. Nilsson. Agent-supported assessment for adaptive and personalized ambient assisted living. In J. Corchado, J. Prez, K. Hallenborg, P. Golinska, and R. Corchuelo, editors, Trends in Practical Applications of Agents and Multiagent Systems, volume 90 of Advances in Intelligent and Soft Computing, pages 25-32. Springer Berlin Heidelberg, 2011.

10. J. Marcais, N. Spanoudakis, and P. Moraitis. Using argumentation for ambient assisted living. In Artificial Intelligence Applications and Innovations, pages 410-419. Springer, 2011.

11. S. Sá and J. Alcântara. Cooperative dialogues with conditional arguments. In Proceedings of the 11th International Conference on Autonomous Agents and Multiagent Systems-Volume 1, pages 501-508. International Foundation for Autonomous Agents and Multiagent Systems, 2012. 